\title{
High Salt Diet Blunted Baroreflex Sensitivity (BRS) to Bilateral Common Carotid Artery Occlusion (BCCO) in Male Sprague -Dawley Rats - Permissive Effect of Testosterone
}

\author{
A. K. Oloyo, S. O. Elias, S. N. Tom, D. C. Ezemadu, K. B. Ismail-Badmus and O. A. Sofola
}

\section{ABSTRACT}

\begin{abstract}
Autonomic dysfunction is involved in the onset of salt-sensitive hypertension as baroreflex sensitivity (BRS) is blunted in salt-sensitive hypertension. However, salt sensitivity exhibits sex disparity because in salt-sensitive hypertension, blood pressure (BP) is usually higher in males when compared with females. The mechanism(s) underlying this sexual dimorphism is not clear. We, therefore, designed this study to determine BRS in weanling male Sprague - Dawley rats that were either bilaterally orchidectomized or sham-operated (under ketamine and xylazine anaesthesia $(90 \mathrm{mg}$ and $10 \mathrm{mg} / \mathrm{kg} / \mathrm{body}$ weight i.m) respectively, with or without testosterone replacement $\left(10 \mathrm{mg} / \mathrm{kg}\right.$ sustanon $250^{\circledR}$ i.m once in 3 weeks) and were placed on normal $(0.3 \%)$ or high $(8 \%) \mathrm{NaCl}$ diet for 6 weeks. Blood pressure (BP) and heart rate (HR) were measured via arterial cannulation under urethane and $\alpha$-chloralose anesthesia $(5 \mathrm{ml} / \mathrm{kg}$ body weight i.p). BRS was determined as change in $H R$ per unit change in BP in response to 30 seconds bilateral common carotid artery occlusion (BCCO). Serum concentration of testosterone was measured using enzyme-linked immunosorbent assay (ELISA). High salt diet (HSD) increased both the basal BP and peak BP after BCCO when compared with control. Orchidectomy attenuated but testosterone replacement restored the elevated BP in rats fed HSD. HSD blunted BRS to BCCO. However, this effect of HSD was lost in the absence of testosterone as there was no significant difference in BRS between rat fed a normal or high salt diet. Our results suggest that blunting of the BRS may be one of the mechanisms by which testosterone promotes salt-induced hypertension and serves as one of the bases for sex disparity in salt-sensitive hypertension.
\end{abstract}

Keywords: Baroreflex sensitivity, Bilateral common carotid occlusion, High salt diet, Hypertension, Testosterone.
Submitted : March 12, 2021

Published : April 19, 2021

ISSN: 2593-8339

DOI: $10.24018 /$ ejmed.2021.3.2.770

\section{A. K. Oloyo*}

Department of Physiology Faculty of Basic Medical Sciences, College of Medicine, University of Lagos, IdiAraba, Lagos, Nigeria.

(e-mail: akoloyo@cmul.edu.ng)

S. O. Elias

Department of Physiology Faculty of Basic Medical Sciences, Lagos State University, College of Medicine, Ikeja, Lagos, Nigeria.

(e-mail: simiat.elias@lasucom.edu.ng) S. N. Tom

Department of Physiology Faculty of Basic Medical Sciences, College of Medicine, University of Lagos, IdiAraba, Lagos, Nigeria.

D. C. Ezemadu

Department of Physiology Faculty of Basic Medical Sciences, College of Medicine, University of Lagos, IdiAraba, Lagos, Nigeria.

K. B. Ismail-Badmus

Department of Physiology Faculty of Basic Medical Sciences, College of Medicine, University of Lagos, IdiAraba, Lagos, Nigeria.

(e-mail: ismailbadmuskb@ gmail.com)

O. A. Sofola

Department of Physiology Faculty of Basic Medical Sciences, College of Medicine, University of Lagos, IdiAraba, Lagos, Nigeria.

(e-mail: oasofola@yahoo.com)

\section{INTRODUCTION}

Dietary salt consumption in high quantity is the commonest environmental risk factor for hypertension [1] as dietary salt intake correlates positively with blood pressure (BP). However, the mechanisms linking salt to hypertension are not well-understood.

For instance, an abnormal baroreflex control of heart rate has been linked to the pathogenesis of essential hypertension [2], [3] and impaired baroreflex sensitivity (BRS) has been demonstrated in salt-sensitive hypertension [4]-[6]. The baroreflex system buffers the changes in BP by the beat-tobeat regulation of the heart rate and peripheral vascular resistance [7]. A sudden increase in BP usually leads to reflex slowing of the heart rate and a reduction in the arterial tone due to enhanced vagal tone and attenuated sympathetic efferent activity [8]. BRS is a measure of the gain of the reflex [8] and factors that reduce BRS include ageing, hypertension, diabetes, and myocardial infarction [9], [10]. However, HSD is reported to reduce or blunt BRS via mechanism involving 
enhanced sympathetic nervous system responses to sympatho-excitatory stimuli [11], [5]. This decreases sympathetic nervous system withdrawal during arterial baroreceptor stimulation and hence inhibit bradycardia [11]. We have previously reported that amlodipine, a calcium channel blocker reversed the BRS blunting effect of HSD [6], suggesting an extra-neural mechanism of baroreflex control.

However, sensitivity of BP to HSD is sex dependent as its adverse effect has been shown to be higher in males when compared with females in human [12] and in animals [13]. The sexual dimorphic nature of BP sensitivity to HSD is attributed to the different sex hormones in male and female. For instance, estrogen has been reported to affect cardiovascular functions via direct actions on the blood vessels and on the autonomic system [14]. Estrogen is suggested to influence baroreflex functions in human [15] and animals [16], [17]. Likewise, Khalid et al. [18] reported that the development of salt-dependent hypertension in male Sabra rats depends on testosterone. We had previously reported that blood pressure elevation by HSD through vascular functions impairment mechanism in male SpragueDawley rats is enhanced by testosterone [19].

Although, dysfunction of autonomic nervous system is implicated in the development of salt-induced hypertension, the role of testosterone in cardiovascular autonomic response to salt-induced hypertension is not clear. Therefore, we designed experiments to study the baroreflex response to bilateral common carotid occlusion (BCCO) as BRS in orchidectomised weanling male Sprague - Dawley rats with or without testosterone replacement and placed on a normal or high salt diet.

\section{MATERIALS AND METHODS}

Eight weeks old weanling male Sprague-Dawley rats were used for this study. The rats weighed between 90 and $110 \mathrm{~g}$ and were kept in cages in our animal care facility at 12 hours light and 12 hours dark period. The rats were provided with food and water ad libitum. We randomly grouped the rats into six with each group containing 6 animals. Groups 1 and II contained rats that were sham-operated, groups III and IV contained rats that were bilaterally orchidectomised while groups V and VI contained rats that were given Sustanon ${ }^{\circledR}$ injection as testosterone replacement post orchidectomy. For orchidectomy, randomly selected rats were anaesthetized with intramuscular $90 \mathrm{mg}$ and $10 \mathrm{mg} / \mathrm{kg} /$ body weight of ketamine and xylazine respectively [20], [21], [24], thereafter their 2scorotums were removed surgically under an aseptic condition. Sham-operated rats of groups I and II rats only had their scrotal sacs opened and sutured back as a model of sham orchidectomy [21]; [19]. At the end of the surgical procedure, all the rats that were operated on were injected intramuscularly with penicillin 300,000 i.u/ $\mathrm{kg}$ body weight. This is to prevent post-operative infections. We allowed all the rats that we operated on a 3-day recovery period before we commenced the study [22], [19]. We fed rats in groups I, III, and IV with chow that contains normal salt concentration $(0.3 \% \mathrm{NaCl})$ and tap water for 6 weeks, while we fed rats in groups II, IV and VI with HSD $(8 \% \mathrm{NaCl})$ and tap water for 6 weeks [23]. As a form of testosterone replacement, we gave rats in groups $\mathrm{V}$ and $\mathrm{VI}$ a $10 \mathrm{mg} / \mathrm{kg}$ body weight dose of testosterone suspension (Sustanon $250^{\circledR}$ i.m) once in 3 weeks during the study. Sustanon $250^{\circledR}$ is a trade name for an oilbased injectable blend of four esterized testosterone compounds. The composition of Sustanon $250^{\circledR}$ are as follows: $30 \mathrm{mg}$ Testosterone Propionate and $60 \mathrm{mg}$ Testosterone Phenylpropionate - a short and fast acting testosterone ester, as well as $60 \mathrm{mg}$ Testosterone Isocaproate, and $100 \mathrm{mg}$ Testosterone Decanoate, both which are slow and long-lasting testosterone esters [21], [24].

\section{A. Measurement of Body and Organs Weight}

We weighed the rats before the commencement of the study and thereafter weekly for the duration of the study. We used a Duet top loading weighing scale (Salter, England) for the weighing of the rats. We calculated the weight gain after the study as:

$$
\frac{\text { final weight }- \text { initial weight }}{\text { initial weight }} \times 100 \%
$$

\section{B. Blood Pressure Measurement}

We measured BP in the rats using arterial cannulation method as described earlier [21]. Briefly, under a solution of $25 \% \mathrm{w} / \mathrm{v}$ urethane and $1 \% \mathrm{w} / \mathrm{v} \alpha$ - chloralose $5 \mathrm{ml} / \mathrm{kg}$ body weight intraperitoneal anaesthesia, the rat femoral artery was cannulated for BP measurement as follows; we inserted into the femoral artery, a polyethylene cannula that was filled with $1 \%$ heparinized saline. We thereafter connect the cannular to the Grass Polygraph recorder (Grass Instruments) via a pressure transducer. The rats were allowed a 15 -minute rest post cannulation to ensure stabilization of the BP readings before the experimental data collection. Blood pressure tracing were obtained as phasic systolic and diastolic pressure from which mean arterial blood pressure was determined as. diastolic pressure $+\frac{1}{3}$ pulse pressure.

\section{Determination of Baroreflex Sensitivity}

Baroreflex sensitivity abnormalities is usually demonstrated with a sudden increase in BP, but it is also demonstrable when blood pressure is lowered for instance with SNP [25]; [26]. In this study, BRS to low-pressure induced by bilateral common carotid occlusion (BCCO) was used. BCCO was carried out by simultaneously applying bulldog clips to both common carotid arteries about $10 \mathrm{~mm}$ below their bifurcation. In each rat, BCCO was carried out thrice at 10 minutes interval with each occlusion lasting for 30 seconds. Heart rate was measured by counting arterial pulse in a 1 - second interval from the polygraph generated $\mathrm{BP}$ tracing and BRS was calculated from measurement of the rate-pressure response to 30 seconds bilateral occlusion of the carotid artery as change in heart rate per unit change in mean arterial blood pressure (beats $/ \mathrm{min} / \mathrm{mmHg}$ ) post BCCO.

\section{Measurement of Urinary Concentration of Sodium}

As an indirect estimation of dietary sodium consumption, we measured the urinary concentration of sodium using a Coring Flame Photometer (Model 410C). Briefly, at room temperature, we thawed the urine samples were retrieved from the freezer. Via a compressed air method, we sprayed 
the diluted urine sample as fine droplets into a luminous gas flame. This luminous gas turned colourful according to the characteristic emission of sodium ions in the sample. The light wavelength was thereafter measured via a light filter and this corresponds to that of the metal being estimated. The amount of light emitted is a representative of the concentration of the ions present in the sample. Using deionized water, we adjusted the flame photometer to zero. Thereafter we prepared a standard sample and dilute other samples with deionized water to 1 in 200 dilutions. We set the standard knob for sodium at $140 \mathrm{mEq} / \mathrm{L}$ and we subject the various sample dilutions to the flame and their concentration recorded.

\section{E. Estimation of Testosterone Concentration in the Serum}

We estimated the concentration of the serum testosterone as previously described [24]. Briefly, we separated the serum from the collected whole blood. Thereafter, the serum was stored at $-80{ }^{\circ} \mathrm{C}$. The concentrations of the serum testosterone were estimated via the enzyme-linked immunoassay method using a commercially available kit from Biotech Laboratories (Suffolk, UK). The estimation was done according to the manufacturer instructions. The estimation mechanism was based on the principle of competitive microplate enzyme immunoassay, whereby testosterone that is present in the sample competes with enzyme-testosterone conjugate for binding with an antitestosterone-coated microplate to form an antigen-antibody complex.

\section{F. Analysis of Data}

We expressed the collected data as mean \pm S.E.M. The collected data were analysed using one - way analysis of variance (ANOVA) firstly, and differences between individual means were detected with Student Newman Keuls as posthoc tests. We placed the confidence interval at $95 \%$ (Graph Pad Prism 5 software).

\section{RESULTS}

\section{A. Body and Organs Weights}

The percent change in the body weight and the organ weight indices of the rats after the six-week experimental period are presented in Table I. The magnitude of weight change in the high salt fed rats were significantly less $(p<0.05)$ when compared with their corresponding control group. HSD significantly increased the heart and kidney weight indices in the animals. Orchidectomy reduced the gain in the cardiac and renal weight that was observed in the HSD group as there was a significant reduction in the cardiac and renal indices of the orchidectomy plus HSD rats when compared with that of the sham plus HSD rats. However, the increase in the heart and kidney weight indices due to HSD was restored by testosterone replacement as the cardiac and renal weight indices of the testosterone replacement plus HSD group were comparable to that of the sham plus HSD group, whereas it was significantly higher $(p<0.05)$ than that of the orchidectomy plus HSD group.
TABLE I: BODY WEIGHTS AND ORGAN INDICES OF RATS THAT WERE ORCHIDECTOMIZED WITH OR WITHOUT TESTOSTERONE REPLACEMENT AND PLACED ON A NORMAL OR HSD

\begin{tabular}{cccc}
\multicolumn{3}{c}{ PLACED ON A NORMAL OR HSD } \\
\hline Groups n = 6 & $\begin{array}{c}\text { \% Increase in } \\
\text { Body Weight }\end{array}$ & Heart Index & \multirow{2}{*}{ Kidney Index } \\
\hline Sham + NS & $48.78 \pm 1.15$ & $0.33 \pm 0.01$ & $0.30 \pm 0.01$ \\
Sham + HS & $36.83 \pm 2.41^{\alpha \alpha}$ & $0.38 \pm 0.01^{\mp}$ & $35 \pm 0.01^{\mp}$ \\
Orch + NS & $51.54 \pm 1.48$ & $0.29 \pm 0.01$ & $0.31 \pm 0.01$ \\
Orch + HS & $47.71 \pm 1.66^{\beta}$ & $0.32 \pm 0.01^{\mu}$ & $0.32 \pm 0.01$ \\
Orch + Tes + NS & $43.76 \pm 3.29$ & $0.32 \pm 0.02$ & $0.31 \pm 0.02$ \\
Orch + Tes + HS & $31.98 \pm 1.71^{\alpha \alpha}$ & $0.36 \pm 0.01^{\mp}$ & $0.33 \pm 0.01$ \\
\hline
\end{tabular}

Data are presented as mean \pm S.E.M, $\mathrm{n}=6$. Significant decrease $(\alpha \alpha \mathrm{p}<0.01)$ when compared with corresponding controls. Significant increase $(\beta p<0.05)$ when compared with Sham plus HSD. Significant increase $(\Phi p<0.05)$ when compared with corresponding control. Significant decrease $(\mu \mathrm{p}<0.05)$ when compared with Sham plus HSD.

\section{B. Basal and Peak Blood Pressure and Heart Rate Parameters}

The basal MABP was significantly higher $(\mathrm{p}<0.05)$ in the HSD groups when compared with their corresponding normal diet group as shown in Table II. The reduction in the MABP of orchidectomy plus normal salt diet group when compared with orchidectomy plus HSD group was interesting. This suggest that even in the absence of a stressor like salt, testosterone plays a role in the normal physiological control of BP. Orchidectomy reduced the elevated BP that was observed in rats fed HSD, as there was a significant reduction $(\mathrm{p}<0.05)$ in the basal MABP of the orchidectomy plus HSD when compared with that of the sham plus HSD group. However, testosterone replacement restored MABP to similar values that was observed in sham plus HSD group. There was also a significant increase $(\mathrm{p}<0.05)$ in the MABP of the testosterone plus HSD group when compared with that of the orchidectomy plus HSD group.

Fig. 1 and 2 show the effect of HSD on the change in MABP and HR in Sprague-Dawley rats with or without testosterone replacement. HSD significantly increased $(\mathrm{p}<0.05)$ the change in MABP and HR when compared with groups fed a normal salt diet. This increase in the magnitude of change in MABP and HR of rats fed HSD were significantly reduced $(\mathrm{p}<0.05)$ by orchidectomy, but testosterone replacement restored the value towards that of the sham orchidectomized rats.

TABLE II: BASAL AND Post-BCO Blood Pressure AND HeART Rate PARAMETERS IN RATS THAT WERE ORCHIDECTOMIZED WITH OR WITHOUT

\begin{tabular}{ccccc}
\multicolumn{4}{c}{ TESTOSTERONE REPLACEMENT AND PLACED ON A HSD } \\
\hline Groups n $=6$ & $\begin{array}{c}\text { Basal } \\
\text { MABP } \\
(\mathrm{mmHg})\end{array}$ & $\begin{array}{c}\text { Peak } \\
\text { MABP } \\
\text { after BCO } \\
(\mathrm{mmHg})\end{array}$ & $\begin{array}{c}\text { Basal } \\
\text { Heart Rate } \\
\text { (beat/min) }\end{array}$ & $\begin{array}{c}\text { Peak Heart } \\
\text { Rate after } \\
\text { BCO } \\
\text { (beat } / \mathrm{min})\end{array}$ \\
\hline Sham + NS & $115 \pm 2$ & $127 \pm 5$ & $390 \pm 13$ & $414 \pm 20$ \\
Sham + HS & $139 \pm 3^{\alpha \alpha}$ & $158 \pm 6^{\alpha \alpha}$ & $408 \pm 8$ & $456 \pm 15$ \\
Orch + NS & $105 \pm 4$ & $109 \pm 4$ & $344 \pm 15$ & $390 \pm 24$ \\
Orch + HS & $\beta^{121} \pm 4^{\alpha \alpha}$ & $\beta 134 \pm 6^{\alpha \alpha}$ & $366 \pm 15$ & $414 \pm 13$ \\
Orch + Tes + NS & $120 \pm 5$ & $126 \pm 5$ & $374 \pm 23$ & $391 \pm 12$ \\
Orch + Tes + HS & $131 \pm 5^{\alpha \alpha}$ & $149 \pm 4^{\alpha \alpha}$ & $396 \pm 18$ & $468 \pm 15$ \\
\hline
\end{tabular}

Data are presented as mean \pm S.E.M, $\mathrm{n}=6$. Significant increase $(\alpha \alpha p<0.01)$ when compare with corresponding controls. Significant increase $(\beta p<0.05)$ when compared with Sham plus HSD. 


\section{Baroreflex Sensitivity to $\mathrm{BCCO}$}

Baroreflex sensitivity to $\mathrm{BCCO}$ of the rats was shown in Fig. 3. HSD significantly $(\mathrm{p}<0.05)$ reduced the BRS to BCCO when compared with the normal salt diet group. The reducing effect of HSD on BRS to BCCO was counteracted by orchidectomy as there exist a significant increase $(p>0.05)$ in BRS to BCCO in orchidectomy plus HSD group when compared with Sham plus HSD group. Likewise, there was no significant difference ( $p>0.05$ ) in BRS to BCCO between sham plus normal salt diet and orchidectomy plus HSD, suggesting that orchidectomy restored BRS to normal in HSD fed group. The reducing effect of HSD on BRS to BCCO was restored by replacement of testosterone in orchidectomized rats. This was evident by a significant reduction in the BRS to $\mathrm{BCCO}$ in testosterone replacement plus HSD group when compared with testosterone replacement plus normal salt diet group. In the same vein, there was a significant reduction in $\mathrm{BRS}$ to $\mathrm{BCCO}$ in testosterone replacement plus HSD group when compared with orchidectomy plus HSD group.

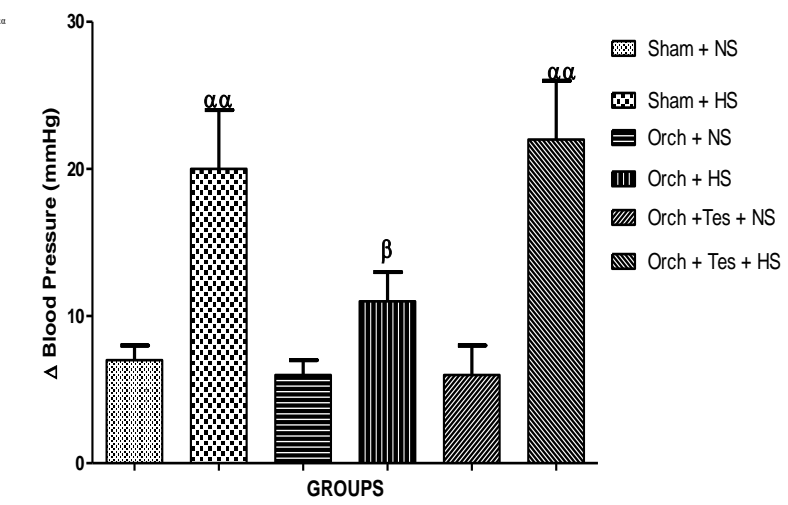

Fig. 1. Effect of HSD on change in MABP after 30 seconds BCCO in orchidectomized Sprague-Dawley rats with or without testosterone replacement. Data are presented as mean \pm S.E.M, $\mathrm{n}=6$. Significant increase $\left({ }^{\alpha \alpha} \mathrm{p}<0.01\right)$ when compared with corresponding controls.

Significant decrease $\left({ }^{\beta} \mathrm{p}<0.05\right)$ when compared with Sham plus HSD.

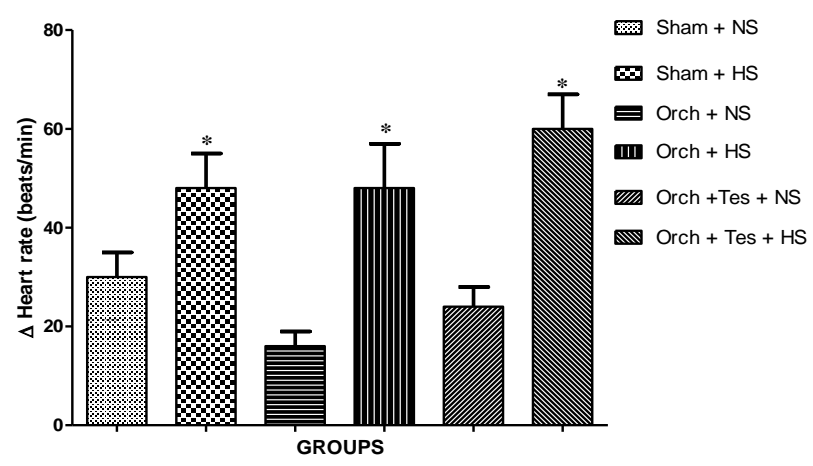

Fig. 2. Change in heart rate after BCO in orchidectomized Sprague-Dawley rats fed a HSD with or without testosterone replacement. Data are presented as mean \pm S.E.M, $n=6$. Significant increase $(* p<0.05)$ when compare with corresponding controls.

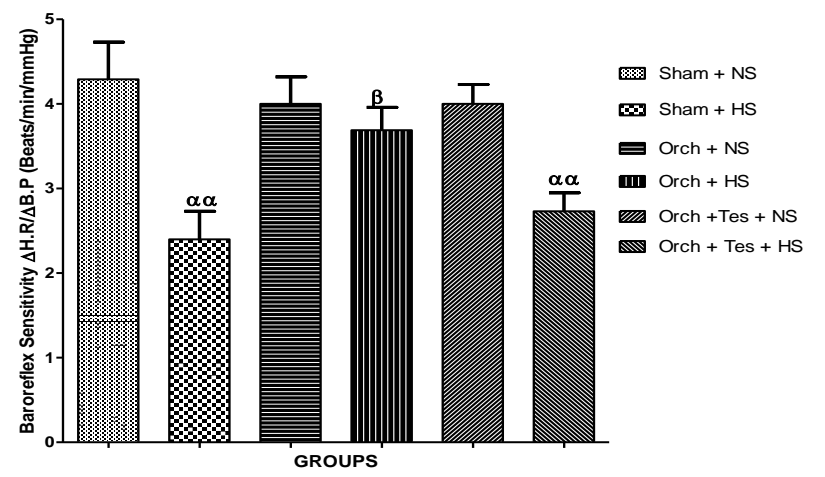

Fig. 3. Baroreflex sensitivity to BCO in orchidectomized Sprague-Dawley rats fed a HSD with or without testosterone replacement. Data are presented as mean \pm S.E.M, $n=6$. Significant decrease $(\alpha \alpha p<0.01)$ when compared with corresponding controls. Significant increase $(\beta p<0.05)$ when compared with Sham plus HSD.

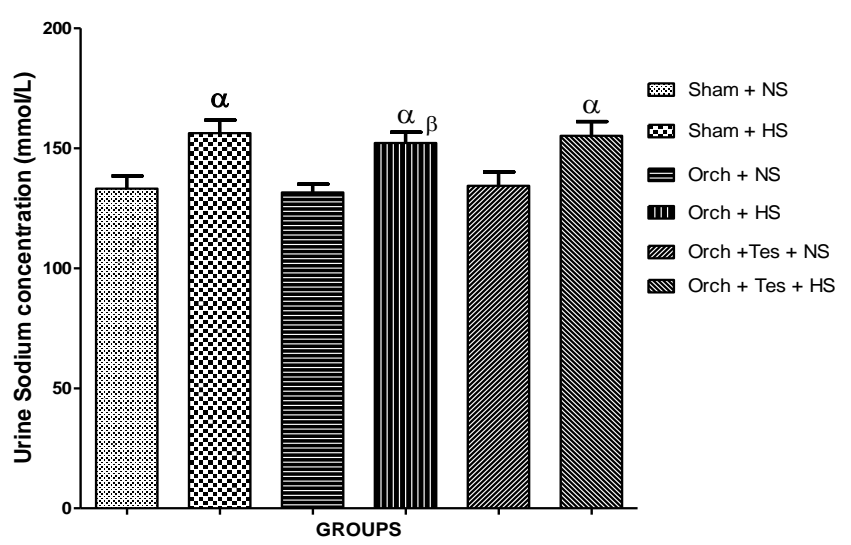

Fig. 4. Effect of HSD on urinary concentration of sodium in orchidectomized Sprague-Dawley rats with or without testosterone replacement. Data are presented as mean \pm S.E.M, $n=6$. Significant decrease $\left({ }^{\alpha} p<0.05\right)$ when compare with corresponding controls. Significant decrease $\left({ }^{\beta} \mathrm{p}<0.05\right)$ when compared with Sham plus HSD.

\section{Concentrations of Sodium in the Urine}

HSD significantly increased $(\mathrm{p}<0.05)$ the concentration of sodium in the urine of groups as shown in Figure 4.

\section{E. Concentration of Testosterone in the Serum}

Figure 5 shows the concentration of testosterone in the serum of the rats. Orchidectomy reduced the serum concentration of testosterone to the barest minimum in both normal and HSD fed rats as there was a significant reduction in the serum concentration of testosterone in orchidectomized rats when compared with both the sham orchidectomized and testosterone replacement group. Testosterone replacement elevated the serum concentration of testosterone towards normal because there was a significant increase in the serum concentration of testosterone in the testosterone replacement groups when compared with the orchidectomy group. There was also no significant difference in serum concentration of testosterone in the testosterone replacement group when compared with that of the sham orchidectomized groups. Interestingly, there was a significant increase $(p<0.05)$ in serum concentration of testosterone in all the rats that were fed HSD when compared with the rats that were fed a normal salt. 


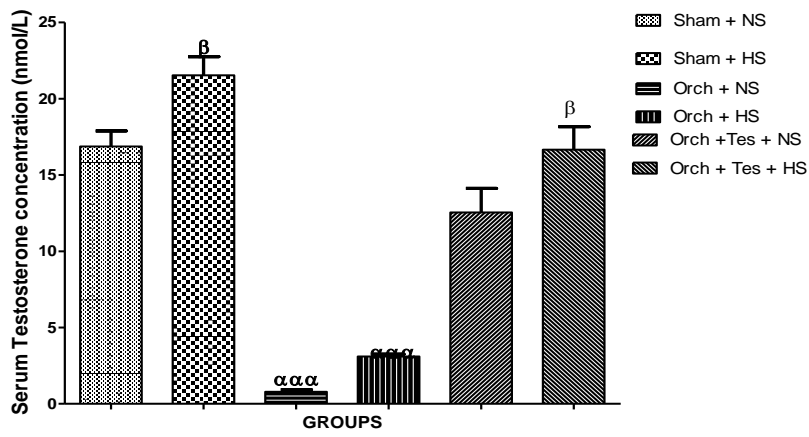

Fig. 5. Serum testosterone concentration of orchidectomized SpragueDawley rats fed a HSD with or without testosterone replacement. Data are presented as mean \pm S.E.M, $n=6$. Significant decrease ( $\left.{ }^{\alpha \alpha a} p<0.001\right)$ when compared with sham operated and testosterone replaced groups. Significant increase $\left({ }^{\beta} \mathrm{p}<0.05\right)$ when compared with corresponding HSD.

\section{DISCUSSION}

The major findings from this study are that:

1) HSD has hypertrophic effect on the heart and kidneys;

2) HSD elevated BP and blunted BRS to BCCO in the male rat;

3) testosterone deprivation through orchidectomy counteracts BP elevating and BRS blunting effects of HSD in the male rat;

4) replacement of testosterone in the orchidectomized rats reestablished BP elevating and BRS blunting effect of HSD.

High intake of dietary salt is an important environmental risk factor for hypertension [1] as studies have shown positive correlation between dietary salt and hypertension [27], [28]. However, the mechanism(s) underlying salt sensitivity is not clear. Suggested mechanisms by which salt elevates BP and causes hypertension include impairment of cardiovascular and renal functions, dysregulation of fluid volume and autonomic system as well as blunting of BRS [6], [29], [30].

The observation in this study that rats loaded with HSD has a decreased percent body weight gain is consistent with previous study. For instance, it has been reported that HSD caused a reduction in percentage body weight gain of rats [31]. It has been shown that HSD increases appetite and therefore food intake. Despite this, there was a reduction in weight gain, a phenomenon that was linked to an increase in body metabolism rate and consequently an increase in energy expenditure, thence a decrease in body weight [31]. The observed decrease in the magnitude of gain in body weight of rats fed a HSD in the present study, can also be related to an increase in body water loss through excessive urination which we observed during the study.

An increase in an organ weight index could indicate swelling or hypertrophy in that organ. Excessive dietary salt intake has been associated with target organ damage, in the presence or absence of elevated BP [30]. The increased organ weight indices (hearts and kidneys) recorded in the rats that were fed HSD, agrees with the previous study that has linked increased dietary sodium to left ventricular hypertrophy [32] and reduced renal function [33].

Our study showed that HSD has a blunting effect on BRS to bilateral common carotid artery occlusion. This is consistent with existing evidence that showed that there is impaired baroreflex sensitivity in response to the increase in plasma volume, following salt loading [25], [34], [6].
Increased dietary sodium affect baroreceptor reflexes by altering the central sympathetic mechanisms of BP regulation. This leads to diminished sympathetic withdrawal and decreased baroreflex buffering of the increased BP in response to salt loading [34]. Therefore, baroreceptors are set to operate at higher pressures in chronic hypertension [35]. However, the abolition and reestablishment of BRS blunting effect HSD by orchidectomy and testosterone replacement respectively indicates a permissive role of testosterone in this process.

Serum testosterone levels were greatly reduced in rats following orchidectomy; and this was restored to normal physiological concentration as it obtains in the sham-operated rats by testosterone replacement. Testosterone replacement restored the BP elevating as well as BRS blunting effects of HSD in rats that underwent orchidectomy procedure. This suggests that testosterone permits the blunting of BRS that is caused by HSD. There appears to be little or no literature on the effect of dietary salt on serum level of testosterone, but the use of dietary salt as aphrodisiac aha been documented by the ancient Greek mythology [36]. Interestingly, dietary salt has also been reported to enhance fertility and improve reproductive functions [36]. Aldosterone has been demonstrated to stimulates the production of testosterone from the Leydig cells [37]. Furthermore, an androgen receptor blocker called flutamide has been shown to increase the level of aldosterone in the serum of orchidectomized rats [38]. Taking all these together, there appears an interaction between dietary salt intake, its main regulatory hormone (aldosterone) and testosterone production and bioavailability. The fact that aldosterone and testosterone are steroidal hormones which apart from sharing the same precursor, are both formed through steroidogenesis further reinforced this suggestion. Wang et al, [39] reported that aldosterone stimulated testosterone production via a mineralocorticoid receptor (MR)-mediated mechanism. Another angle to look at the marginal elevation in the serum concentration of testosterone in rats given HSD is the fact that, both HSD and chronic exposure to testosterone elevates or heightens the vascular tone [40].

However, our findings are also in contrast with previous reports that suggests that testosterone has cardioprotective properties. In a study carried out by El-Mas et al [41], testosterone facilitates baroreceptor control through the enhancement of cardiac efferent vagal activity. Testosterone supplementation has also been reported to induce an increase in BRS in patients suffering from chronic heart failure [42], [43]. These observed differences in experimental outcomes could be due to prevailing underlying pathologies and the mechanism(s) by which testosterone modulates them. We studied BRS to BCCO in HSD-fed rats while El-Mas and colleagues [41] studied role of testosterone in BRS under non-physiological condition. Caminiti, Yoshihisa and their colleagues reported the role of testosterone in existing pathologies such as insulin insensitivity and heart failure in aged male participants [42], [43].

Although, the role of testosterone in promoting and/or attenuating hypertension and cardiovascular diseases remain unclear, our present findings suggest that blunting of the baroreflex sensitivity to BCCO could be involved in the mechanisms by which testosterone promotes salt-induced 
hypertension and this may explain the sex disparity that exist in sensitivity of BP to HSD.

\section{ACKNOWLEDGEMENT}

This Study Was Funded By The University Of Lagos Central Research Grant (Unilag Crc Research Grant) 2007/14.

\section{REFERENCES}

[1] P. Meneton, X. Jeunemaitre, H. E. de Wardener, \& G. A. Macgregor. (2005). Links between dietary salt intake, renal salt handling, blood pressure, and cardiovascular diseases. Physiological reviews. 2005.

[2] R. J. Parmer, J. H. Cervenka, \& R. A. Stone. Baroreflex sensitivity and heredity in essential hypertension. Circulation, 85(2), 497-503. 1992.

[3] D. Hering, A. Trzebski, \& K. Narkiewicz. Recent advances in the pathophysiology of arterial hypertension: potential implications for clinical practice. Pol Arch Intern Med, 127(3), 195-204. 2017.

[4] B. Özaykan, \& A. Doğan. Effects of salt loading on sympathetic activity and blood pressure in anesthetized two-kidney, one clip hypertensive rats. Bosnian Journal of Basic Medical Sciences, 11(4), 228. 2011.

[5] P. Castiglioni, G. Parati, D. Lazzeroni, M. Bini, A. Faini, L. Brambilla, ... \& P. Coruzzi. Hemodynamic and autonomic response to different salt intakes in normotensive individuals. Journal of the American Heart Association, 5(8), e003736. 2016.

[6] A. A. Adejare, \& O. A. Sofola. Amlodipine Corrects Changes in Blood Pressure and Baroreceptor Reflex Sensitivity in Sprague Dawley Rats Fed a High Salt Diet. Nigerian Journal of Physiological Sciences, 32(1), 63-67. 2017.

[7] A. J. Broadley, A. Korszun, E. Abdelaal, V. Moskvina, C. J. Jones, G. B. Nash, ... \& M. P. Frenneaux. Inhibition of cortisol production with metyrapone prevents mental stress-induced endothelial dysfunction and baroreflex impairment. Journal of the American College of Cardiology, 46(2), 344-350. 2005.

[8] E. A. Wehrwein, \& M. J. Joyner. Regulation of blood pressure by the arterial baroreflex and autonomic nervous system. In Handbook of clinical neurology (Vol. 117, pp. 89-102). 2013.

[9] N. Nasr, A, Pavy-Le Traon, \& V. Larrue. Baroreflex sensitivity is impaired in bilateral carotid atherosclerosis. Stroke, 36(9), 1891-1895. 2005.

[10] M. T. La Rovere, G. D. Pinna, \& G. Raczak. Baroreflex sensitivity: measurement and clinical implications. Annals of noninvasive electrocardiology, 13(2), 191-207. 2008.

[11] S. L. Bealer. Increased dietary sodium inhibits baroreflex-induced bradycardia during acute sodium loading. American Journal of Physiology-Regulatory, Integrative and Comparative Physiology, 288(5), R1211-R1219. 2005.

[12] M. Bursztyn, \& I. Z. Ben-Dov. Sex differences in salt-sensitivity risk approximated from ambulatory blood pressure monitoring and mortality. Journal of hypertension, 31(5), 900-905. 2013.

[13] C. Hinojosa-Laborde, T, Craig, W. Zheng, H. Ji, J. R. Haywood, \& K. Sandberg. Ovariectomy augments hypertension in aging female Dahl salt-sensitive rats. Hypertension, 44(4), 405-409. 2004.

[14] V. L. Brooks, P. A. Cassaglia, D. Zhao, \& R. K. Goldman. Baroreflex function in females: changes with the reproductive cycle and pregnancy. Gender medicine, 9(2), 61-67. 2012.

[15] B. E. Hunt, J. A. Taylor, J. W. Hamner, M. Gagnon, \& L. A. Lipsitz. Estrogen replacement therapy improves baroreflex regulation of vascular sympathetic outflow in postmenopausal women. Circulation, 103(24), 2909-2914. 2001.

[16] M. M. el-Mas , A. A. Abdel-Rahman. Estrogen enhances baroreflex control of heart rate in conscious ovariectomized rats. Can J Physiol Pharmacol. 76(4): 381-6. 1998.

[17] J. Pamidimukkala, J. A. Taylor, W. V. Welshons, D.B. Lubahn, \& M. Hay. Estrogen modulation of baroreflex function in conscious mice. American Journal of Physiology-Regulatory, Integrative and Comparative Physiology, 284(4), R983-R989. 2003.

[18] M. Khalid, N. Ilhami, Y. Giudicelli, and J. P. Dausse. Testosterone dependence of salt-induced hypertension in Sabra rats and role of renal $\alpha$ (2)-adrenoceptor subtypes. J Pharmacol Exp Ther 300:43-49. 2002.

[19] A. K. Oloyo, O. A. Sofola, \& M.A. Yakubu. Orchidectomy attenuates high-salt diet-induced increases in blood pressure, renovascular resistance, and hind limb vascular dysfunction: role of testosterone.
Clinical and Experimental Pharmacology and Physiology, 43(9), 825833. 2016

[20] R. J. Gonzales, D. N. Krause, S. P. Duckles. Testosterone suppresses endothelium-dependent dilation of rat middle cerebral arteries. Am. J. Physiol. Heart Circ. Physiol. 286(2): H552-H560. 2004.

[21] A. K. Oloyo, O. A. Sofola, \& C. N. Anigbogu. Orchidectomy attenuates impaired endothelial effects of a high-salt diet in Sprague-Dawley rats. Canadian journal of physiology and pharmacology, 89(4), 295-304. 2011.

[22] J. Zhu, M. Yu, J. Friesema, T. Huang, R. J. Roman, J. H. Lombard Salt-induced ANG II suppression impairs the response of cerebral artery smooth muscle cells to prostacyclin. Am. J. Physiol. Heart Circ. Physiol. 288(2): H908-H913. 2005.

[23] B. J. Adegunloye, \& O. A. Sofola. Effect of dietary salt loading and high-calcium diet on vascular smooth muscle responses and endothelium function in rats. Clinical and experimental pharmacology and physiology, 24(11), 814-818. 1997.

[24] A. K. Oloyo, O. A. Sofola, C. N. Anigbogu, R. R. Nair, H. S. Vijayakumar, \& A. C. Fernandez. Testosterone reduces vascular relaxation by altering cyclic adenosine monophosphate pathway and potassium channel activation in male Sprague Dawley rats fed a highsalt diet. Therapeutic Advances in Cardiovascular Disease, 7(2), 7585. 2013.

[25] E. Miyajima, \& R. D. Bunag. Chronic cerebroventricular infusion of hypertonic sodium chloride in rats reduces hypothalamic sympathoinhibition and elevates blood pressure. Circulation research, 54(5), 566-575. 1984

[26] F. J. He, \& G. A. MacGregor. Plasma sodium and hypertension. Kidney international, 66(6), 2454-2466. 2004.

[27] S. K. Ha. Dietary salt intake and hypertension. Electrolytes \& Blood Pressure, 12(1), 7-18. 2014.

[28] Q. Zhao, D. Gu, J. Chen, L. A. Bazzano, D. C. Rao, J. E. Hixson, C. E. Jaquish, J. Cao, J. Chen, J. Li, T. Rice, J. He. Correlation Between Blood Pressure Responses to Dietary Sodium and Potassium Intervention in a Chinese Population, American Journal of Hypertension, Volume 22, Issue 12(1), 1281-1286. 2009.

[29] T. A. Kotchen, A. W. Cowley, \& E. D. Frohlich. Salt in health and disease - a delicate balance. New England Journal of Medicine, 368(13), 1229-1237. 2013.

[30] W. B. Farquhar, D. G. Edwards, C. T. Jurkovitz, \& W. S. Weintraub. Dietary sodium and health: more than just blood pressure. Journal of the American College of Cardiology, 65(10), 1042-1050. 2015.

[31] M. S. Coêlho, M. D. Passadore, A. L. Gasparetti, T. Bibancos, P. O. Prada, L. L. Furukawa, ... \& J. Luz. High-or low-salt diet from weaning to adulthood: effect on body weight, food intake and energy balance in rats. Nutrition, metabolism and cardiovascular diseases, 16(2), 148155. 2006.

[32] Y. Jin, T. Kuznetsova, M. Maillard, T. Richart, L. Thijs, M. Bochud, .. \& J. A. Staessen. Independent relations of left ventricular structure with the 24-hour urinary excretion of sodium and aldosterone. Hypertension, 54(3), 489-495. 2009.

[33] H. J. Heerspink, G. Navis, \& E. Ritz. Salt intake in kidney disease-a missed therapeutic opportunity? Nephrology Dialysis Transplantation, 27(9), 3435-3442.2012.

[34] A. Grillo, S. Lucia, C. Paolo, S. Paolo, and P. Gianfrancoi. "Sodium intake and hypertension." Nutrients 11, no. 9 (2019): 1970.

[35] V. M. A. Farah, E.D. Moreira, M. C. Irigoyen, \& E.M. Krieger Baroreflex depression persists in the early phase after hypertension reversal. American Journal of Physiology-Regulatory, Integrative and Comparative Physiology, 280(6), R1620-R1626. 2001.

[36] B. M. Moinier and T. B. Drueke (2008). Aphrodite, sex and salt - From butterfly to man. Nephrol Dial Transplant 23: $2154-2161$.

[37] R. S. Ge, Q. Dong, C. M. Sottas, S. A. Latif, D.J. Morris, M. P. Hardy MP. Stimulation of testosterone production in rat Leydig cells by aldosterone is mineralocorticoid receptor mediated. Mol Cell Endocrinology. 24; 243 (1-2): 35 - 42. 2005.

[38] P. J. Hoffman, M. Michaelis, F. Gotz, C. Bartel, T. Kienitz, M Quinkler. Flutamide increases aldosterone levels in gonadectomised male but not female Wistar rats. Am J Hypertens 25(6): 697 - 703 2012.

[39] Y. Wang, H. Li, Q. Zhu, X. Li, Z. Lin, \& R. S. Ge. The cross talk of adrenal and Leydig cell steroids in Leydig cells. The Journal of steroid biochemistry and molecular biology, 192, 105386. 2019.

[40] T. Kienitz, \& M. Quinkler. Testosterone and blood pressure regulation. Kidney and Blood Pressure Research, 31(2), 71-79. 2008.

[41] M. M. El-Mas, E. A. Afify, M. M. M. El-Din, A. G. Omar, \& F. M. Sharabi. Testosterone facilitates the baroreceptor control of reflex bradycardia: role of cardiac sympathetic and parasympathetic components. Journal of cardiovascular pharmacology, 38(5), 754-763. 2001 . 
[42] G. Caminiti, M. Volterrani, F. Iellamo, G. Marazzi, R. Massaro, M. Miceli, ... \& G. M. Rosano. Effect of long-acting testosterone treatment on functional exercise capacity, skeletal muscle performance, insulin resistance, and baroreflex sensitivity in elderly patients with chronic heart failure: a double-blind, placebo-controlled, randomized study. Journal of the American College of Cardiology, 54(10), 919-927. 2009.

[43] A. Yoshihisa, S. Suzuki, Y. Sato, Y. Kanno, S. Abe, M. Miyata, ... \& Y. Takeishi, Y. Relation of testosterone levels to mortality in men with heart failure. The American journal of cardiology, 121(11), 1321-1327. 2018. 